ÉGYPTE

monde arabe

\section{Égypte/Monde arabe}

12-13 | 1993

Une économie en transition

\title{
L'économie de l'énergie en Égypte : la rente en question
}

\section{Louis Blin}

\section{(2) OpenEdition}

1 Journals

Édition électronique

URL : https://journals.openedition.org/ema/1243

DOI : 10.4000/ema.1243

ISSN : 2090-7273

\section{Éditeur}

CEDEJ - Centre d'études et de documentation économiques juridiques et sociales

\section{Édition imprimée}

Date de publication : 31 mars 1993

Pagination : 21-47

ISSN : 1110-5097

\section{Référence électronique}

Louis Blin, «L'économie de l'énergie en Égypte : la rente en question », Égypte/Monde arabe [En ligne], 12-13 | 1993, mis en ligne le 08 juillet 2008, consulté le 07 juillet 2022. URL : http:// journals.openedition.org/ema/1243; DOI : https://doi.org/10.4000/ema.1243

Ce document a été généré automatiquement le 7 juillet 2022.

Tous droits réservés 


\title{
L'économie de l'énergie en Égypte : la rente en question
}

\author{
Louis Blin
}

L'énergie, traditionnelle clé du développement économique, est également en Égypte un important pourvoyeur de devises. Ceci met en relief le caractère délicat des années à venir, qui devraient voir la diminution de la rente pétrolière dont jouit ce pays. L'avenir énergétique de l'Égypte dépend en grande partie de sa croissance démographique et de son développement économique, particulièrement de l'évolution de l'intensité énergétique du produit intérieur brut. L'alignement à l'horizon 1995 des prix intérieurs de l'énergie sur les prix mondiaux - auquel le gouvernement égyptien s'est engagé vis-à-vis de ses créditeurs internationaux - est crucial à cet égard, car il permettrait de mettre fin au gaspillage de l'énergie et, partant, de réduire l'augmentation de la consommation intérieure. L'économie de l'énergie en Égypte traverse actuellement une période de transition caractérisée par l'importance décroissante des exportations de pétrole et le coût accru de l'extraction du pétrole, qui annoncent la fin de l'ère de la rente pétrolière telle qu'elle a marqué le pays depuis 1976. Ceci ne signifie pas l'assèchement du sous-sol égyptien, donc la disparition de la rente pétrolière, mais une transformation progressive de la nature de cette dernière, d'externe à interne : l'économie de l'énergie en Égypte, ou la rente en question.

Tableau 1 : Bilan du secteur des hydrocarbures

Image1

2 Cet article n'aborde que l'économie du secteur énergétique égyptien en tant que tel (hydrocarbures et électricité), laissant donc de côté son rôle dans d'autres domaines de l'économie, ses activités hors d'Égypte (prestations de services notamment), ses relations extérieures (structure du commerce extérieur énergétique de l'Égypte) et sa place sur la scène énergétique mondiale, ainsi que le transit énergétique par l'Égypte (par l'oléoduc Sumed et le canal de Suez). De même que les transferts des émigrés, les recettes générées par ce transit sont liées à l'économie de l'énergie régionale, et non égyptienne. 


\section{Les hydrocarbures}

3 L'Égypte est un vieux pays pétrolier - puisque l'exploration remonte à 1884 et la production à 1911 -, un producteur moyen, au seizième rang mondial avec 53,1 millions de tonnes en 1991, gaz compris (tableau 1), et un exportateur de taille non négligeable avec $20 \mathrm{Mt} / \mathrm{an}$ ces dernières années, enlèvements des compagnies inclus (l'Égypte est exportatrice nette depuis 1976).

\section{Des réserves médiocres}

Le niveau annuel moyen des découvertes des dernières années s'élève à 50.000 barils/ jour de pétrole et 40 millions de pieds cubes de gaz naturel, y compris les découvertes jugées non commerciales (le jugement en la matière pouvant varier en fonction du prix du baril, de la proximité d'autres découvertes et de révolution des techniques d'extraction). Les découvertes récentes les plus importantes, surtout des gisements de gaz naturel, ont permis de renouveler les réserves. Comme criez tous les producteurs, il faut se méfier des dates prévues d'épuisement des réserves, qui n'ont de valeur que relative. Le pétrole existe, il suffit de le trouver, puis de l'exploiter. II faut pour cela s'en donner les moyens, grâce à la promotion d'une industrie locale et à la coopération internationale, et que les prix mondiaux du pétrole soient suffisamment élevés pour justifier cette activité, paramètre échappant aux autorités égyptiennes.

5 En matière pétrolière, l'installation d'équipements de récupération assistée et la mise en production de nouveaux champs visent à compenser la baisse prévisible de la production des gisements anciens du golfe de Suez. Les réserves pétrolières de l'Égypte n'ont cessé de progresser ces dernières années, passant de 2,9 milliards de barils en 1982 à 4,6 milliards de barils en 1991, soit une durée de vie de 14,7 ans au rythme de production actuel. Cette estimation est sans doute exagérée, le ministre du Pétrole ayant indiqué en mars 1992 une espérance de vie des réserves pétrolières de 10-12 ans seulement. L'expérience de la prospection pétrolière en Égypte rend improbable la présence de grands champs aux coûts d'exploitation susceptibles de se rapprocher de ceux des pays du Golfe. Les nombreuses découvertes de gisements commerciaux dans les années 80 et l'état de l'exploration du pays (les accords de prospection ne couvrent actuellement que $25 \%$ du territoire) permettent d'infirmer les prévisions pessimistes qui rangent l'Égypte parmi les pays importateurs de pétrole dans cinq à six ans. Cependant, compte tenu de la part du pétrole produit que l'État doit reverser aux compagnies en vertu des accords de partage de la production, l'Égypte pourrait commencer à acheter du pétrole dans huit ans environ.

Dans le domaine du gaz naturel, les réserves ont été réévaluées en 1991 à 12 milliards de pieds cubes, contre 6,9 Gpc en 1985, ce qui donne une durée de vie de 40 ans. Ce chiffre a peu de valeur analytique puisque la production est appelée à augmenter de quelque $8 \%$ par an, mais que l'exploration a été moins poussée pour le gaz que pour le pétrole jusqu'à présent. Les dernières estimations du ministre du Pétrole indiquent 31 ans de réserves. Selon la Banque mondiale, la consommation dépasserait l'offre d'environ $400 \mathrm{Mpc} / \mathrm{j}$ en 1995 et de 600 à $700 \mathrm{Mpc} / \mathrm{j}$ en l'an 2000 si les seules réserves identifiées sont développées. La découverte d'importants gisements gaziers dans des régions non productrices est toutefois possible, et l'objectif gouvernemental est de 
porter les réserves à $27.000 \mathrm{Gpc}$ pour couvrir la demande intérieure au cours des vingt années à venir.

\section{L'exploration : des conditions attractives pour les compagnies étrangères}

7 Après un ralentissement au milieu des années 80, l'activité exploratrice des sociétés étrangères en Égypte a progressivement repris à partir de 1987 pour les raisons suivantes :

1. l'entrée en vigueur en 1987 d'une «clause gaz » incitatrice a stimulé la prospection. Le gaz découvert ne donnait pas jusqu'alors lieu à partage de la production, ce qui dissuadait l'exploitation des champs de gaz et même du gaz associé. La clause gaz aligne pour l'essentiel la juridiction gazière sur celle relative au pétrole, de plus longues périodes étant toutefois consenties pour les phases d'exploration et d'exploitation. Le monopole public Egyptian General Petroleum Corporation (EGPC) rachète la part de la société de production dans le cadre de contrats à long terme à un prix équivalent à $85 \%$ de celui du fuel oil ;

2. l'unification du marché des changes en 1991 a entraîné une baisse de $35 \%$ du taux de change appliqué aux dépenses en monnaie locale des sociétés pétrolières ;

3. la fixation en 1991 d'un nouveau mode de calcul des prix à l'exportation des pétroles égyptiens a amélioré les conditions de vente ;

4. les relations avec les autorités sont satisfaisantes, le secteur des hydrocarbures échappant largement aux problèmes bureaucratiques connus par les autres secteurs économiques. Le changement de ministre du Pétrole en 1991 n'a pas modifié les orientations gouvernementales, ce qui a été apprécié par les compagnies, très attachées à la stabilité ;

5. illustration récente de ces bonnes relations: l'assouplissement en cours de la législation pétrolière. Les derniers appels d'offres pour l'exploration de nouveaux blocs envisagent pour la prospection dans des zones difficiles des améliorations au cas par cas des accords de partage de la production actuels, qui prévoient le prélèvement de $20 \%$ de la production pour la récupération des coûts d'exploration et de développement et le partage des $80 \%$ restants entre l'EGPC (85\%) et son partenaire (15\%), laissant à l'Égypte une part de $68 \%$ du total de la production. Les règles de la clause gaz sont également négociables.

8 Ces facteurs expliquent l'activité soutenue de recherche pétrolière en Égypte :

$9-172$ accords d'exploration ont été signés depuis 1973, dont 75 conclus avec 50 sociétés étaient en vigueur en mars 1992. 20 de ces sociétés sont productrices de pétrole. Ces accords ont mené à 126 découvertes commerciales, qui ont produit 5,4 milliards de barils, dont 4 milliards dans le seul golfe de Suez. Le montant des dépenses d'exploration est passé de 228 à 300 millions \$ entre 1990 et 1991, et il a totalisé 15 milliards $\$$ au cours des 20 dernières années ;

10 - le nombre de puits forés a progressé de 62 en 1987 à 70 en 1991. 45 équipes de recherche sismique étaient en activité en 1989 ;

11 - une diversification géographique de la recherche pétrolière s'opère depuis 1989. Aux zones traditionnelles (golfe de Suez, puis Désert occidental plus récemment) sont venues s'adjoindre des régions non productrices : confins des frontières libyennes, nord du Sinaï, partie off-shore du Delta du Nil, Haute-Égypte et, cette année, permis au large du Désert occidental et nouveaux blocs proposés le long de la côte de la mer Rouge jusqu'au Soudan ; 
12 - un frein à l'exploration (deux accords renégociés en 1991) est cependant apparu ces deux dernières années, les promoteurs touristiques prétendant interdire la recherche en certains points du littoral de la mer Rouge, notamment dans la région de Hurghada, en raison du boom du tourisme balnéaire. Leurs arguments étant basés sur le respect de l'environnement, les ministres du Pétrole et de l'Environnement ont signé en février 1992 un protocole régissant l'exploration pétrolière en matière environnementale, qui délimite des zones protégées et édicté un code de conduite des sociétés pétrolières. Cinq centres de contrôle pétrolier ont par ailleurs été installés en divers points du littoral pour surveiller plus particulièrement les tankers transitant par les eaux égyptiennes.

Tableau 2 : Évolution prévue du secteur des hydrocarbures (milliers de tonnes)

\begin{tabular}{|c|c|c|c|c|c|c|c|}
\hline . & $1992-93$ & $1993-94$ & 1994-95 & $1995-96$ & 1996-97 & $1997-98$ & $1998-99$ \\
\hline Production & & & & & & & \\
\hline Brut (a) & 43.500 & 41.760 & 40.090 & 38.486 & 36.947 & 35.469 & 34.050 \\
\hline Gaz naturel & 11.736 & 12.423 & 12.375 & 12.329 & 12.282 & 14.284 & 15.598 \\
\hline Condensats,' GPL & 3.525 & 3.719 & 3.705 & 3.372 & 3.755 & 4.291 & $4.670^{\circ}$ \\
\hline Total & 58.761 & 57.902 & 56.170 & 54.547 & 52.984 & 54.044 & 54.318 \\
\hline \multicolumn{8}{|l|}{ Rattinage, Consommation } \\
\hline Entrées en raffinerie & 23.772 & 26.184 & 26.196 & 26.189 & 26.193 & 26.038 & 25.906 \\
\hline Production des raffineries & 23.486 & 25.836 & 25.836 & 25.836 & 25.836 & 25.836 & 25.836 \\
\hline Consommation (b) : & 17.426 & 16.881 & 17.095 & 17.398 & 18.109 & 18.654 & 19.186 \\
\hline (b) $/$ (a) & $40,1 \%$ & $40,4 \%$ & $42,6 \%$ & $45.2 \%$ & $49,0 \%$ & $52,6 \%$ & $56.3 \%$ \\
\hline Consommation de gaz naturel & 12.338 & 13.015 & 12.967 & 13.062 & 13.015 & 15.016 & 16.341 \\
\hline \multicolumn{8}{|l|}{ Commerce extérieur (net) } \\
\hline Brut & 19.728 & 15.576 & 13.894 & 12.297 & 10.754 & 9.431 & 8.144 \\
\hline Produits pétroliers & 5.795 & 8.702 & 8.485 & 8.175 & 7.435 & 6.867 & 6.313 \\
\hline Total & 25.523 & 24.278 & 22.379 & 20.472 & 18.189 & 16.298 & 14.457 \\
\hline
\end{tabular}

Source : Banque mondiale, mars 1992.

\section{La production : stabilité ou déclin?}

13 La production égyptienne d'hydrocarbures (pétrole brut, gaz naturel, condensats et GPL) est passée de 34,6 millions de tonnes équivalent pétrole en 1982 à un record de 53,1 Mtep en 1991 (tableau 1), augmentation due au maintien d'une forte production de pétrole et à la croissance rapide de la production de gaz (multipliée par 3,3 entre ces deux dates). La Banque mondiale prévoit la poursuite de cette augmentation jusqu'en 1992-93 (58,8 Mt), puis un lent déclin dû à une chute de la production de brut, que n'arriveront pas à compenser les hausses des productions de gaz naturel, GPL et condensats (tableau 2).

14 La production de pétrole brut s'est élevée à 875.000 barils/jour en 1991, en ligne avec l'objectif de $870.000 \mathrm{~b} / \mathrm{j}$ ( $44 \mathrm{Mt} / \mathrm{an}$ ) fixé par le plan 1987-88/1991-92, repris par le plan 1992-93/1996-97 (cf. EMA 10, p. 206). Cet objectif parait irréaliste, les principaux gisements étant désormais sur le déclin. La production de brut devrait en fait baisser régulièrement jusqu'à $34 \mathrm{Mt}$ en 1998-99, selon la Banque mondiale. La principale région 
productrice de pétrole en Égypte est le golfe de Suez avec $63 \%$ de la production en 1990-91, suivi du Sinaï avec 25,3 \% et du reste du pays (Désert occidental surtout) avec $11,6 \%$. La part de cette région va croissant, puisqu'elle ne représentait que $8,9 \%$ en 1987-88.

La production de gaz naturel a connu un accroissement annuel moyen de $15 \%$ depuis 1984, qui l'a porté au niveau record de 8,3 Mtep en 1991-92, dont $30 \%$ de gaz associé à la production de pétrole. Elle devrait passer à 15,6 Mtep en 1996-97 (+88 \%) grâce à l'exploitation de nouveaux champs, qui entraînerait par ailleurs la multiplication par trois de la production de GPL et de condensats entre 1991 (1,6 Mt) et 1998-99 (4,7 Mt). La production de gaz naturel provient principalement du Delta du Nil (68,2\% de la production totale en 1990-91), suivi par le golfe de Suez (17,4\%), le Désert occidental $(13,4 \%)$ et le Sinaï $(1,0 \%)$. La production de gaz est donc fortement concentrée dans les zones densément peuplées, ce qui facilite son utilisation. Les seules régions productrices d'hydrocarbures, sont donc pour le moment le golfe de Suez et le nord du pays.

La production de produits raffinés est passée de 14,3 Mt en 1981 à 23,8 Mt en 1991 et la capacité théorique de raffinage atteignait $29,4 \mathrm{Mt}$ à la mi-1992. Bien que les projets en cours soient supposés porter cette capacité à $35,35 \mathrm{Mt} / \mathrm{an}$, la production devrait plafonner à 25,8 Mt entre 1994 et la fin du siècle $(+8,5 \%)$, selon la Banque mondiale. L'Égypte dispose de sept raffineries à Suez (deux raffineries de $100.000 \mathrm{~b} / \mathrm{j}$ et $58.000 \mathrm{~b} / \mathrm{j}$, en cours d'extension à $240.000 \mathrm{~b} / \mathrm{j}$ au total), Ameriya $(75.000 \mathrm{~b} / \mathrm{j})$, Tanta $(25.000 \mathrm{~b} / \mathrm{j}$, en cours d'extension à; $38.000 \mathrm{~b} / \mathrm{j})$, Mostorod $(115.000 \mathrm{~b} / \mathrm{j}$, en cours d'extension à $170.000 \mathrm{~b} / \mathrm{j})$, Alexandrie $(115.000 \mathrm{~b} / \mathrm{j})$ et Assiout $(50.000 \mathrm{~b} / \mathrm{j}$, en cours de doublement). Elles sont de configuration simple avec peu de capacités secondaires de traitement pour transformer le fuel lourd en distillais et pour augmenter l'indice d'octane, ce qui conduit l'Égypte à exporter du fuel lourd et du naphta, mais à importer du kérosène pour l'aviation et du gas oil. L'EGPC a mis à l'étude un programme de modernisation des raffineries pour les adapter au profil de la consommation, le gouvernement ayant décidé de relever de 5 degrés l'indice d'octane des carburants (actuellement 90 pour le super) et d'introduire l'essence sans plomb au Caire et à Alexandrie.

\section{La concurrence entre consommation et exportations}

17 La production de pétrole brut est passée de 32,1 millions de tonnes en 1982 à 44,7 Mt en 1991, tandis que la consommation de produits raffinés progressait de 16,3 Mt à 20,1 Mt durant la même période. Le rapport entre les deux a donc baissé entre ces deux dates de $50,6 \%$ à $44,9 \%$. En tenant compte des erreurs statistiques et de la balance importations/exportations de produits raffinés, il apparaît qu'environ la moitié de la production pétrolière est actuellement consommée sur place, l'autre moitié étant exportée. Aucune exportation de gaz n'est jamais intervenue. En l'absence de nouvelle découverte pétrolière majeure, les exportations pétrolières nettes (brut + produits) devraient passer par un maximum de 25,5 Mt en 1992-93, grâce au remplacement du fuel par le gaz naturel dans les centrales électriques, puis décliner jusqu'à 14,5 Mt en 1998-99.

18 Une politique de maîtrise de la consommation de pétrole a été engagée il y a dix ans. Le rythme annuel de progression de la consommation de produits pétroliers et de gaz naturel a fléchi de $11,2 \%$ entre 1981 et 1985 à $4 \%$ de 1985 à 1989, mais davantage en 
raison des difficultés économiques que grâce aux économies d'énergie. Au cours des dernières années, le gaz a par ailleurs concouru autant que le pétrole à l'augmentation de la consommation. Après une hausse de $23,4 \%$ entre 1982 et 1991, la consommation de produits pétroliers devrait stagner en deçà de 20 Mt jusqu'en 1998-99, notamment grâce à la hausse de la consommation de gaz naturel, qui doublerait entre 1990-91 (8,15 Mtep) et 1998-99 (16,3 Mtep). Le plan 1991-92/1996-97 estime cependant que la consommation de produits raffinés progressera entre ces deux dates de 20,4 à 22,5 Mt, soit un taux annuel de croissance de $4 \%$.

Ce n'est que sous l'impulsion du FMI que le gouvernement a entrepris de lutter contre le gaspillage engendré par les bas prix de l'énergie: en 1990, la moitié des factures énergétiques restaient impayées et le prix moyen des produits pétroliers représentait $30 \%$ des prix mondiaux. Signé à l'issue de longues négociations n'ayant abouti qu'après la chute des recettes d'exportations pétrolières de l'Égypte, survenue en 1986, l'accord conclu en mai 1987 avec le FMI prévoyait déjà le relèvement des prix intérieurs de l'énergie, mais les autorités égyptiennes l'ont rapidement abandonné. En septembre suivant, l'US AID suspendait tout financement de nouveaux projets dans le domaine énergétique en Égypte tant que le gouvernement de ce pays n'augmenterait pas les tarifs de l'électricité. Les négociations pour un nouvel accord avec le FMI ont notamment achoppé sur les questions énergétiques, les subventions implicites de l'État à l'énergie représentant encore 9,6\% du PIB en 1990-91, selon la Banque mondiale. Augmentés à partir de mai 1991, quelques jours avant le nouvel accord de confirmation avec le Fonds, les prix moyens pondérés des produits pétroliers sur le marché égyptien seront portés d'ici juin 1995, suivant cet accord, à l'équivalent des prix industriels internationaux, soit la moyenne annuelle des prix fob à la sortie des raffineries italiennes, et les prix du gaz naturel vendu aux industriels devront être alignés sur ceux du fuel sur le marché intérieur. Il faut remarquer que cet alignement sur les prix industriels mondiaux ne constitue pas un but en soi, mais un objectif minimal de "vérité des prix ", en portant les tarifs intérieurs au niveau des prix à l'exportation, que l'État égyptien sera par la suite libre de dépasser s'il veut taxer plus avant la consommation d'énergie, comme le font la plupart des pays développés.

La substitution du gaz au pétrole est par ailleurs encouragée dans l'industrie (chimie, ciment et, dans une moindre mesure, engrais) et la génération d'électricité. Après avoir remisé en 1986 leurs projets nucléaires et face aux limites de l'hydro-électricité, les autorités égyptiennes ont opté pour la combustion de gaz dans les centrales électriques nouvelles, équipées de systèmes de récupération de chaleur («cycle combiné ») pour améliorer leur rendement, et la conversion des centrales au fuel en centrales fuel/gaz. Renforcée par diverses améliorations techniques, cette politique a permis de porter le rendement (rapport entre énergies consommée et restituée sous forme d'électricité) des centrales électriques égyptiennes de $26 \%$ en $1982-83$ à $33,6 \%$ en $1990-91.61 \%$ du gaz consommé en Égypte sont par ailleurs utilisés pour la génération d'électricité, $35 \%$ par l'industrie, $3 \%$ par le secteur pétrolier et $1 \%$ par les ménages. Cette substitution rencontre cependant certaines limites, dans la mesure où $75 \%$ de la part des sociétés dans la production de gaz doivent leur être rachetés par le gouvernement égyptien à un prix fixé par rapport à celui du fuel. En pratique, ce rachat s'effectue sous forme de livraisons de pétrole brut aux compagnies, ce qui diminue d'autant le surplus exportable. Des évaluations non officielles font état d'une perte de recettes en devises correspondantes de 213 millions \$ en 1989 et 295 millions \$ en 1990. 
Tableau 3 : Les hydrocarbures dans l'économie égyptienne

\begin{tabular}{|c|c|c|c|c|c|c|c|c|c|c|c|}
\hline 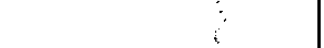 & $1981-82$ & 1982.83 & $1983-84$ & $1984-85$ & $1985-86$ & $1986-87$ & $1987-88$ & $1988-89$ & 1989.90 & $1990-91$ & $1991-92$ \\
\hline Exportations de petrole (M $\$$ ) & 4.669 & 4.164 & 4.532 & 4.781 & 3.995 & 1.225 & 1.385 & 1.148 & 1.229 & 1.971 & 1.545 \\
\hline Part dans les exportations totales & $80,8 \%$ & $79,3 \%$ & $76,5 \%$ & $78,7 \%$ & $76,9 \%$ & $47,4 \%$ & $44,7 \%$ & $40,7 \%$ & $39.1 \%$ & $50.7 \%$ & $43,9 \%$ \\
\hline Part dans les recettes en devises (1) & $44,8 \%$ & $34.5 \%$ & $33,5 \%$ & $36.0 \%$ & $34,0 \%$ & $11,1 \%$ & $11.5 \%$ & $9,2 \%$ & $8,7 \%$ & $10,3 \%$ & $8,7 \%$ \\
\hline Exportations non pastrolières (MS) & 1.110 & 1.084 & 1.392 & 1.294 & 1.198 & 1.360 & 1.713 & 1.672 & 1.915 & 1.916 & 1.978 \\
\hline Contribut & & & & & & & & & & & \\
\hline (M LE) & 1.110 & 891 & 1.066 & 936 & 1.093 & 805 & 911 & 1.033 & 782 & 3.236 & $3.830^{*}$ \\
\hline Part dans les recettes budgétaires (2) & $16,8 \%$ & $11,4 \%$ & $13,0 \%$ & $10,8 \%$ & $11,2 \%$ & $8,4 \%$ & $7,9 \%$ & $7,6 \%$ & $5,0 \%$ & $13,7 \%$ & $11,1 \% *$ \\
\hline Déticit budgétaire (M LE) & 4.883 & 4.748 & 6.433 & 7.165 & 8.844 & 7.738 & 10.849 & 11.847 & 14.518 & 19.779 & 8.700 \\
\hline
\end{tabular}

(1) Exportations, services (canal de Suez, tourisme, etc.), transferts publics et privés.

(2) Recettes réalisées du budget de l'Etat à l'exclusion de celles des collectivités locales, du secteur public et des investissements autofinancés (selon la nomenclaure buogétaire égyptienne) et hars secteur de la défense, non budgétisé.

- Estimations.

Sources : Banque mondiaie jusqu'en 1985.86. FMl ensuite.

\section{D'une rente externe à une rente interne}

21

ex des hydrocarbures dans les pays exportateurs se limite le plus souvent à comptabiliser leurs recettes d'exportations pétrolières, alors que ce qui leur importe le plus, ce sont leurs revenus pétroliers, soit l'excédent qu'ils tirent de l'exploitation de leur pétrole, une fois les dépenses afférentes défalquées. Aussi curieux que cela puisse paraître dans une industrie où l'information est si développée, il n'existe aucun recueil statistique sur ces revenus, qu'on appelle traditionnellement rente pétrolière, vu leur nature.

Mesurer la rente pétrolière d'un pays à l'aune de ses recettes d'exportation est une erreur qui va en s'aggravant à mesure que s'amenuise la différence entre les prix à l'exportation et les coûts d'extraction. Ces coûts ne sont pas négligeables en Égypte parce que les gisements y sont petits, ce qui implique de rémunérer suffisamment les sociétés pétrolières pour qu'elles ne délaissent pas le pays. De plus, la transformation croissante des hydrocarbures avant leur exportation (raffinage, liquéfaction du gaz) leur procure une valeur ajoutée qui altère leur nature rentière. Ce facteur est peu important en Égypte, bien que ses exportations annuelles moyennes de produits pétroliers aient représenté de 1986-87 à 1990-91 31\% des exportations pétrolières totales. En effet, ce pays exporte surtout des produits raffinés peu élaborés (naphta, huile de soute), dont le prix moyen au cours de cette période n'a en conséquence dépassé que de. $15 \%$ les prix moyens à l'exportation de ses bruts.

Bien que l'analyse de l'évolution des recettes d'exportations soit donc insuffisante pour évaluer la place dés hydrocarbures dans un pays exportateur, l'examen de leur part dans ses exportations globales et dans le total de ses recettes en devises donne des indications importantes à ce sujet, notamment pour suivre leur évolution. Le tableau 3 et le graphique 1 mettent en évidence l'ampleur de la rente pétrolière égyptienne jusqu'au "contre-choc » pétrolier de 1986, qui a entraîné une chute de $69,3 \%$ des recettes d'exportations de pétrole entre 1985-86 et 1986-87, puis son lent déclin jusqu'au «mini-choc » de 1990, qui a permis une croissance éphémère de ces recettes (+60,4\% entre 1989-90 et 1990-91). La part des hydrocarbures dans les recettes d'exportations est restée majoritaire jusqu'à la chute des prix du pétrole, puis ce dernier est demeuré le premier produit d'exportation de l'Égypte.

Égypte/Monde arabe, 12-13| 1993 
Le graphique 2 montre, d'une part, que les exportations non-pétrolières, qui stagnaient tant que le pétrole prédominait, ont progressé avec son déclin. Cette coïncidence, qui ne peut être tenue pour fortuite, montre qu'en Égypte comme dans d'autres pays pétroliers, une rente pétrolière importante entrave les exportations non-pétrolières. Elle indique, d'autre part, que l'appareil productif égyptien s'est adapté rapidement au déclin de la rente, mais de manière insuffisante : si les exportations non-pétrolières ont progressé de 78,1\% entre 1981-82 et 1991-92, les exportations globales ont toutefois baissé de $39 \%$ entre ces deux dates. Au vu de l'évolution de la composition de ses exportations, l'Égypte ne peut donc plus être considérée comme un pays pétro-rentier depuis 1986-87, année qui a marqué la fin de la dominance de la rente pétrolière.

\section{Graphique 1}

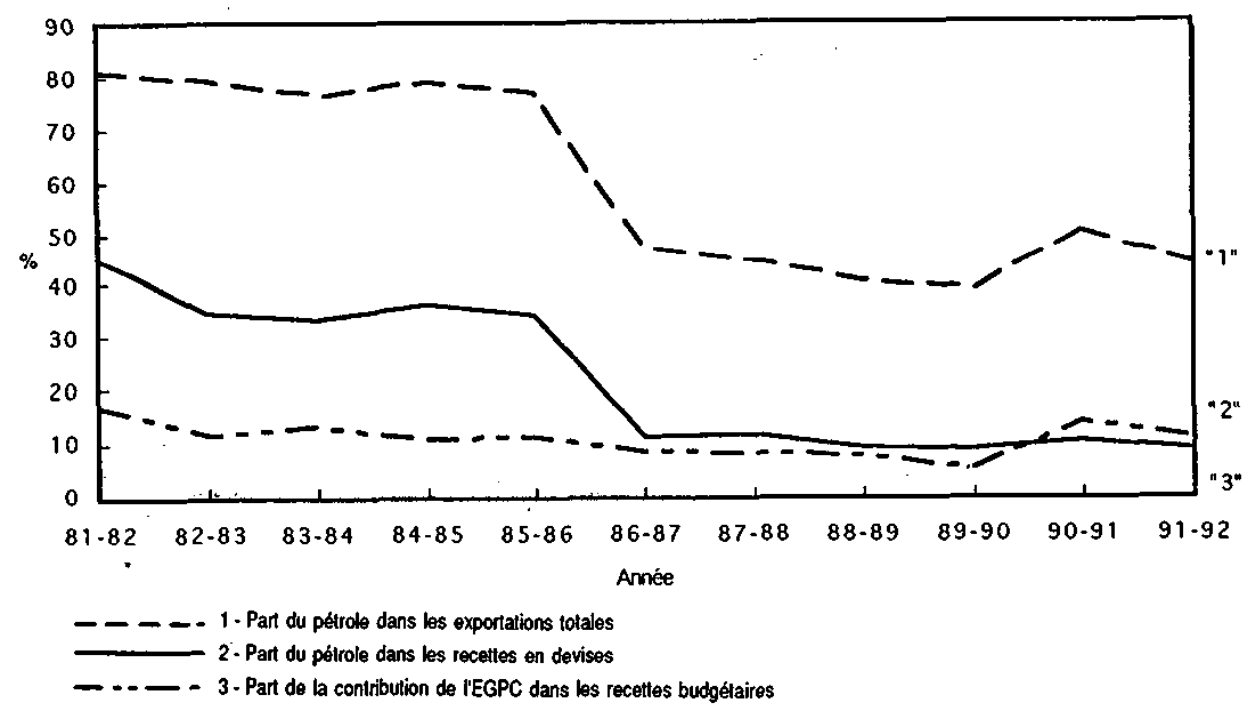

Les courbes (1) et (2) du graphique 1 montrent par ailleurs l'évolution parallèle de la part des hydrocarbures dans les recettes d'exportation et dans l'ensemble des recettes en devises, qui prouve la dépendance de ces dernières à l'égard des prix du pétrole. Comme indiqué en introduction, le pétrole égyptien n'est que la composante interne de l'influence du pétrole sur l'économie de l'Égypte. Il faut y ajouter une rente pétrolière régionale liée à la position géographique de ce pays, qui dépasse le cadre de cet article.

Une autre méthode d'évaluation des revenus pétroliers d'un pays exportateur est de comptabiliser la fiscalité pétrolière inscrite aux budgets annuels. Elle n'est cependant connue qu'une fois établies les réalisations budgétaires, avec retard donc. Les chiffres figurant par ailleurs en monnaie locale, l'examen du budget ne permet pas de calculer la part de cette fiscalité pétrolière perçue en devises, alors qu'une des fonctions essentielles du secteur pétrolier est son rôle de pourvoyeur de devises. La courbe (3) du graphique 1 montre que l'érosion de la place des hydrocarbures dans les recettes budgétaires est plus lente que celle de leur part des recettes en devises, ce qui indique que l'État a comblé en partie la baisse de ses rentrées fiscales externes par une hausse de la taxation des ventes de l'EGPC en Égypte. Malgré cela, la part de la contribution de l'EGPC au budget de l'État a été divisée par 3,4 entre 1981-82 et 1989-90, ce qui n'a pas été pour rien dans la hausse du déficit budgétaire, multiplié par 3 durant cette période (tableau 3). Après une année de transition, qui voit un nouveau dérapage budgétaire 
malgré une fiscalité pétrolière dopée par la crise du Golfe, la réduction du déficit budgétaire entreprise en 1991-92 suivant les désirs du FMI est notamment permise par une hausse de la contribution de l'EGPC au budget, grâce au relèvement des prix de l'énergie en Égypte. Basé sur une estimation constante - et basse - de cette contribution ( $40 \%$ du chiffre d'affaires de cette société), le graphique 3 compare la chute de la fiscalité pétrolière à laquelle on assisterait sans cette hausse des prix, à sa progression dans le cas inverse, d'autant plus forte qu'est renforcé l'usage du gaz naturel, puisque ce dernier est entièrement consommé sur place. Vu que les revenus tirés par l'État de la taxation des hydrocarbures commercialisés sur le marché intérieur augmentent et que la quantité de pétrole exporté tend à la baisse, une rente interne est appelée à se substituer à la rente pétrolière externe.

\section{Graphique 2}

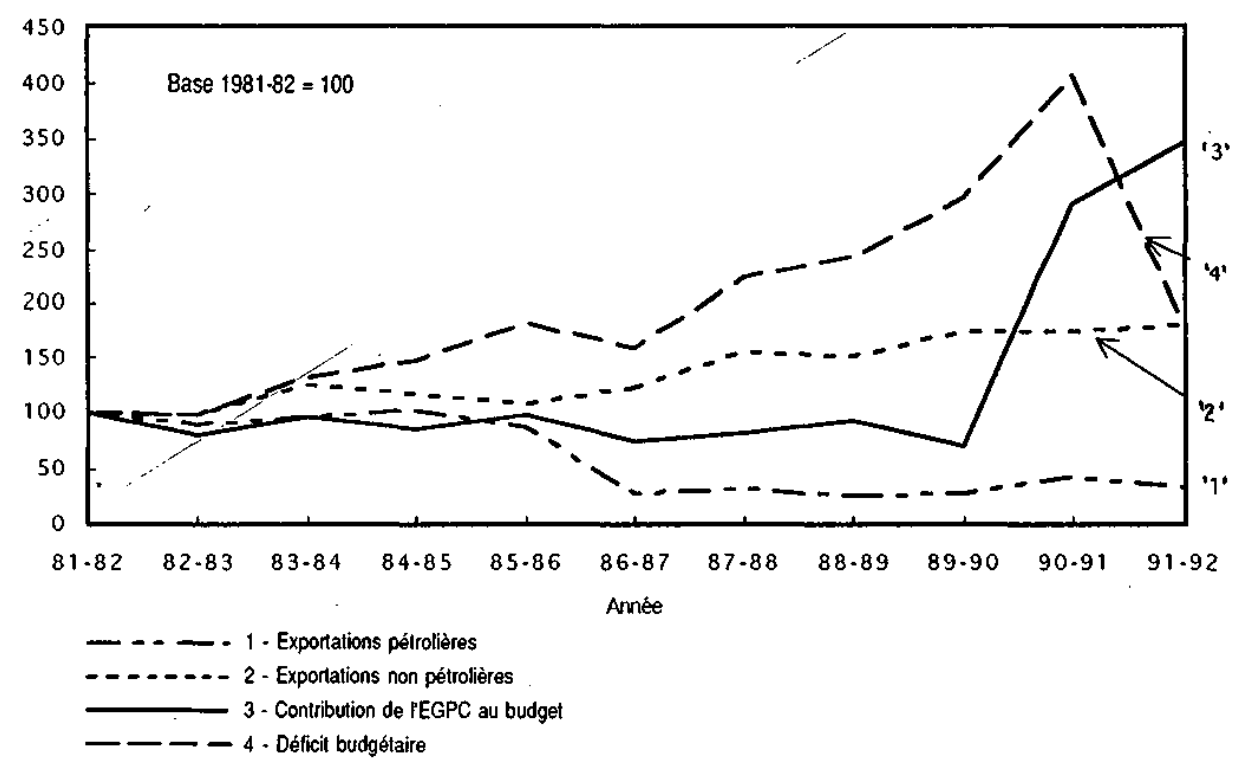


Graphique 3 : Contribution de l'EGPC au budget de l'État (limitée à $40 \%$ du chiffre d'affaires)

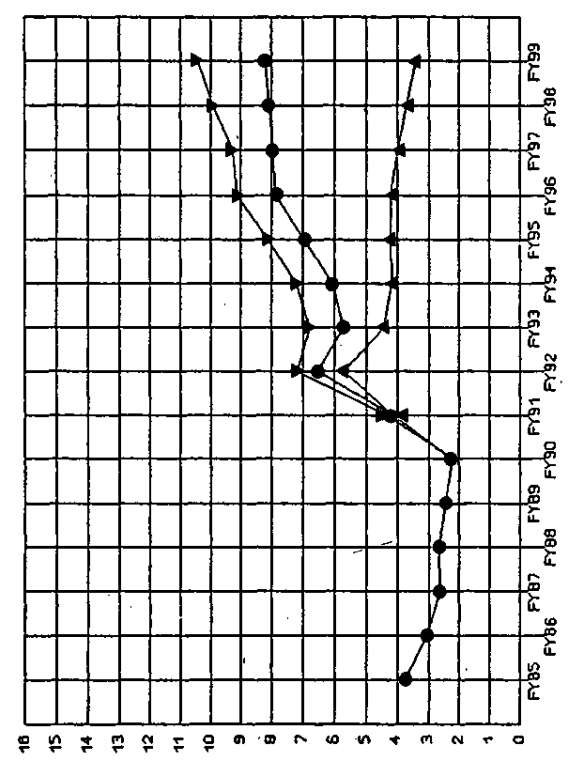

Ordonnée : milliards LE 1990-91

Abcisse : FY85 = année 1984-85 ; FY86 = année 1985-86, et ainsi de suite.

A statu quo

- réforme des prix de lénergie

$\nabla$ réforme el hausse de la consommation de gaz

Pour mesurer cette évolution, il convient de calculer au préalable cette rente externe, c'est-à-dire l'excédent en devises tiré du pétrole, opération complexe. L'excédent pétrolier en devises de l'Égypte s'obtient en effet en additionnant les exportations de brut correspondant à sa part de production, les exportations de produits raffinés, la différence entre le plafond de déduction admis et les dépenses réellement engagées par les compagnies et les bonus de signature, puis en soustrayant les importations de brut (il s'agit en fait du rachat aux compagnies d'une partie du pétrole brut), les importations de produits raffinés et l'achat aux compagnies d'au moins $75 \%$ de leur part de production de gaz. Il doit donc être distingué de l'excédent commercial pétrolier, seule statistique publiée par l'EGPC, qui ne mesure que la différence en valeur entre les hydrocarbures exportés et importés par l'Égypte. Représentant environ $40 \%$ des recettes d'exportation de pétrole, l'excédent pétrolier de l'Égypte est passé de 715 millions \$ en 1988 à 1.356 millions \$ en 1990, pour retomber à 1.312 millions \$ en 1991 (tableau 1), variations liées à celles des cours du pétrole.

Cette instabilité accroît les incertitudes quant à l'évolution du solde pétrolier, basée sur les disponibilités physiques évoquées plus haut. Des prévisions effectuées sur la base d'un prix moyen du baril de $20 \$$ de 1991 à 1995 ou augmentant de façon régulière jusqu'à 25 \$ en 1996, toutes choses égales par ailleurs, font état d'un excédent pétrolier chutant à 1,1 milliard \$ en 1996 dans le premier cas, allant jusqu'à 1,6 milliard \$ dans le second. L'Égypte doit donc envisager à moyen terme une réduction progressive de son excédent pétrolier, à moins d'une envolée des prix du pétrole improbable durant les prochaines années. Le paramètre interne essentiel pour retarder ce déclin est la maîtrise de la consommation pétrolière.

Le plan 1992-93/1996-97 prévoit 9.059 millions \$ d'investissements dans le secteur des hydrocarbures et dérivés, dont 5.721 millions \$ d'investissements étrangers affectés à 
l'exploration/production du pétrole et du gaz, soit $63 \%$ du total, et le solde, soit 11 milliards LE au taux de change de l'année de base, représentant 7,6\% des investissements planifiés, destinés au raffinage, à la distribution et à la pétrochimie. Ce dernier chiffre représente l'équivalent d'environ trois années de surplus pétrolier (converti au même taux et selon le scénario bas), ce qui indique que le secteur des hydrocarbures absorberait durant cette période $60 \%$ des revenus externes qu'il générerait, signe du coût croissant de l'extraction et surtout de la distribution. L'augmentation des revenus internes du secteur, grâce au relèvement des prix au consommateur, représente donc également à terme une condition de l'entretien de ses infrastructures propres. En tant que secteur de souveraineté, les hydrocarbures resteront largement à l'écart de la politique égyptienne de privatisations. Seul l'aval pétrolier sera concerné, mais pas les raffineries, quoique le secteur privé soit autorisé à en construire de nouvelles. L'investissement privé dans la distribution de produits pétroliers est également encouragé. Seules deux entreprises pétrolières publiques doivent être privatisées, sans qu'aucune échéance n'ait pour le moment été fixée, les sociétés de distribution Misr Petroleum Company et Egyptian Petroleum Cooperative Society. Les autorités encouragent enfin les capitaux locaux à s'engager dans l'exploration/production, ce qui s'est traduit par l'attribution, en 1993, de deux premiers permis de recherche à des sociétés privées égyptiennes.

\section{L'électricité}

L'Égypte est l'un des pays du tiers monde les mieux équipés en matière d'électricité. Entre 1981-82 et 1990-91, la capacité de génération et la production d'électricité ont été multipliées par 2,3 et 2, passant de $4.970 \mathrm{MW}$ à $11.435 \mathrm{MW}$ et de $21.899 \mathrm{GWh}$ à 44.145 GWh, respectivement (tableau 4). La forte progression de la consommation d'électricité enregistrée ces dernières années est due à l'accroissement démographique, à l'extension du réseau de distribution, au développement industriel et au gâchis d'énergie engendré par des prix largement inférieurs aux prix mondiaux. En 1988-89, les prix du fuel et du gaz livrés aux centrales représentaient $7,2 \%$ et $8,8 \%$ des prix internationaux, respectivement. Les tarifs de l'électricité ne représentaient au deuxième semestre 1992 que $57,9 \%$ des coûts de production pour les prix au détail $(9,5-$ piastres/Kwh, contre 16,4 piastres/Kwh) et $37,2 \%$ pour le haut voltage (6,1 piastres/ Kwh). Le relèvement des prix de l'énergie prévu dans le cadre du programme d'ajustement structurel devrait permettre d'abaisser le taux de croissance de la consommation d'électricité, les prix de l'électricité devant couvrir les coûts de production marginaux à long terme d'ici juin 1995.

Comme mentionné plus haut, la structure de la consommation d'énergie commerciale en Égypte devrait par ailleurs poursuivre l'évolution amorcée il y a dix ans, qui a vu la part du gaz naturel doubler au détriment de l'hydro-électricité et des produits raffinés, dont la contribution à satisfaire les besoins énergétiques égyptiens baisse depuis 1982-83 et 1985-86, respectivement (tableau 5).

\section{Génération : le gaz tend à supplanter le pétrole}

La production des centrales électriques égyptiennes se répartissait en 1990-91 entre les turbines hydro-électriques ( $9.500 \mathrm{GWh}$, soit $21,5 \%$ du total, contre $72 \%$ en 1974), les 
turbines à vapeur (fuel ou fuel/gaz, $30.967 \mathrm{GWh}, 70,2 \%$ ) et les turbines à gaz (3.678 GWh, 8,3 \%).

Tableau 4 : Bilan du secteur électrique

\begin{tabular}{|l|r|r|r|r|r|r|r|r|r|r|}
\hline & $1981-82$ & $1982-83$ & $1983-84$ & $1984-85$ & $1985-86$ & $1986-87$ & $1987-88$ & $1988-89$ & $1989-90$ & $1990-91$ \\
\hline Capacilé installée (MW) & 4.970 & 5.641 & 6.519 & 7.640 & 8.597 & 8.897 & 9.177 & 10.151 & 10.811 & 11.655 \\
Production d'électricité (GWh) & 21.899 & 24.564 & 27.829 & 30.250 & 32.377 & 35.350 & 38.055 & 39.804 & 41.884 & 44.145 \\
dont : Hydro-électricité & 10.475 & 10.267 & 9.637 & 9.004 & 9.046 & 9.105 & 8.259 & 9.323 & 9.972 & 9.500 \\
$\quad$ Turbines vapeur & 7.965 & 9.410 & 11.968 & 14.424 & 18.042 & 20.406 & 24.282 & 25.095 & 24.098 & 30.710 \\
$\quad$ Tubines gaz & 3.459 & 4.867 & 6.136 & 6.706 & 5.158 & 5.692 & 5.306 & 5.168 & 7.578 & 3.678 \\
$\quad$ Centrales isolées & - & 20 & 88 & 116 & 131 & 147 & 208 & 218 & 236 & 257 \\
Consommation de gaz nalurel & & & & & & & & & & \\
par les centrales (Mm $\left.{ }^{3}\right)$ & 913 & 1.302 & 1.650 & 2.117 & 3.409 & 3.587 & 4.092 & 4.364 & 4.645 & 4.227 \\
\hline
\end{tabular}

Source : Egyptian Electricity Authonity.

Tableau 5 : Évolution de la consommation d'énergie commerciale (milliers de tonnes équivalent pétrole)

\begin{tabular}{|c|c|c|c|c|c|c|c|}
\hline & \multicolumn{2}{|c|}{ Prodults raffinés } & \multicolumn{2}{|c|}{ Gaz naturel } & \multicolumn{2}{|c|}{ Hydro-électricité } & \multirow{2}{*}{ Total } \\
\hline & Quantité & $\%$ & Quantité & $\%$ & Quantité & $\%$ & \\
\hline 1960 & 4.700 & 98,5 & - & - & 70 & 1,5 & 4.770 \\
\hline 1970 & 5.900 & 81,5 & - & $\cdot$ & 1.340 & 18,5 & 7.240 \\
\hline 1980 & 11.700 & 71,8 & 1.800 & 11,0 & 2.800 & $\cdot 17,2$ & 16.300 \\
\hline $1981-82$ & 13.700 & 72,7 & 2.140 & 11,4 & 2.994 & 15.9 & 18.834 \\
\hline 1982.83 & 15.600 & 74,5 & 2.415 & $\$ 1,5$ & 2.935 & 14,0 & 20.950 \\
\hline $1983-84$ & 17.211 & 75,1 & 2.955 & 12,9 & 2.755 & 12,0 & 22.921 \\
\hline $1984-85$ & 18.427 & 75.25 & 3.487 & 14,25 & 2.574 & 10,5 & 24.488 \\
\hline $1985-86$ & 17.746 & 71,0 & 4.661 & 18,65 & 2.586 & 10,35 & 24.993 \\
\hline $1986-87$ & 18.994 & 71,5 & 4.955 & 18.7 & 2.603 & 9,8 & 26.552 \\
\hline $1987-88$ & 19.515 & 70.7 & 5.719 & 20,7 & 2.361 & 8,6 & 27.595 \\
\hline 1988.89 & 19.234 & 68.7 & 6.111 & 21,8 & 2.665 & 9,5 & 28.010 \\
\hline $1989-90$ & 19.892 & 67,5 & 6.705 & 22,8 & 2.851 & 9.7 & 29.448 \\
\hline $1990-91$ & 20.325 & 66,8 & 7.333 & 24,1 & 2.782 & 9,1 & 30.440 \\
\hline
\end{tabular}

Source : Egyptian Electricity Authority.

La production d'hydro-électricité augmentera peu d'ici la fin du siècle $(+4,2 \%$ grâce à l'achèvement du nouveau barrage d'Esna), atteignant $9.900 \mathrm{GWh}$ contre un potentiel théorique de $12.000 \mathrm{GWh} / \mathrm{an}$. Sa part dans le total devrait chuter en conséquence à $10 \%$ du total en 2005, contre $65 \%$ en 1970. La capacité installée du haut-barrage d'Assouan (2.100 MW) n'était utilisée qu'à $57 \%$ en juin 1990 en raison de la sécheresse, le niveau des eaux dans le lac de retenue ayant même menacé d'empêcher son fonctionnement en 1988, mais le réservoir s'est rempli depuis. D'autres centrales ont été construites sur le Nil à Assouan $(345+270 \mathrm{MW})$, Esna $(90 \mathrm{MW})$, Naga Hammadi $(56,5 \mathrm{MW})$ et Assiout (40 MW), ces trois dernières n'étant pas intégrées au réseau de l'Égyptian Electricity Authority (EEA, monopole public). La construction d'un nouveau barrage à Naga 
Hammadi, qui serait doté d'une centrale hydro-électrique de $58 \mathrm{MW}$, vient d'être mise à l'étude. Les projets d'Aïn Sukhna (600 MW) et de la dépression de Qattara (1.800 MW) sont irréalisables dans un avenir proche. La centrale d'Aïn Sukhna, dont l'étude a été confiée en avril 1992 à la firme américaine Ebasco Overseas, repose sur une idée originale : pomper de l'eau de mer aux heures creuses du réseau électrique jusqu'à un réservoir naturel situé dans les monts Galala, qui dominent la côte à cet endroit, et installer une chute d'eau actionnant des turbines en contrebas aux heures de pointe. La centrale de Qattara fonctionnerait à l'eau de mer acheminée par un canal de $160 \mathrm{~km}$ de long qui serait creusé entre la Méditerranée et cette région, située à $135 \mathrm{~m}$ au-dessous du niveau de la mer. La consommation d'électricité d'origine hydraulique pourrait dépasser la production vers la fin du siècle si l'interconnexion électrique égyptozaïroise est réalisée d'ici là (voir ci-après), permettant à l'Égypte d'importer de l'électricité. L'EEA estime que le Zaïre pourrait lui fournir $10.000 \mathrm{GWh} /$ an d'électricité en 2015.

34 L'intention du gouvernement égyptien est d'utiliser au maximum le gaz naturel au détriment des produits pétroliers. La construction du réseau de gazoducs nécessaire à cet effet est facilitée par la concentration de la population sur une superficie restreinte, le recours aux centrales à diesel étant limité aux rares zones d'habitat isolé (les centrales non interconnectées au réseau unifié de l'EEA ne représentaient, avec une production de $257 \mathrm{GWh}$ en 1990-91, que 0,6\% de la production totale d'électricité). La part des centrales à turbines à gaz (y compris cycle combiné) dans la production d'électricité est passée de 15,8\% en 1981-82 à 18,1 \% en 1989-90 et devrait progresser à $26,3 \%$ en 1998-99, et les centrales vapeur sont progressivement équipées pour la bicombustion fuel/gaz, le prix du gaz livré aux centrales étant désormais lié à celui du fuel. $80 \%$ de la puissance installée devraient pouvoir fonctionner au gaz en 1994, contre $65 \%$ actuellement. $51 \%$ de l'électricité produite à partir de sources d'énergie fossiles l'ont été à partir du gaz en 1989-90, contre $25 \%$ dix ans plus tôt, le gaz ayant alors pour la première fois dépassé le fuel dans ce secteur. Ce pourcentage est passé à $53,9 \%$ en 1990-91.

La mise en service de la première centrale électrique égyptienne fonctionnant au charbon, qui serait importée d'Australie, est prévue pour 1997-98 à Ayoun Moussa (650 MW), sur la côte nord-ouest du Sinaï. Une autre centrale du même type est en projet depuis plusieurs années à Zaafarana $(2.400 \mathrm{MW})$ sur la rive occidentale du golfe de Suez. Le charbon devant être extrait de Maghara (Sinaï) à partir de 1993 sera destiné à la sidérurgie.

La catastrophe de Tchernobyl en 1986 a fourni un argument idéal pour reporter sine die l'ambitieux programme de construction de centrales nucléaires envisagé il y a dix ans, en plein boom pétrolier. Le paradoxe du nucléaire dans le tiers monde est qu'il nécessite de considérables moyens financiers que ne détiennent en gros que les pays exportateurs de pétrole, donc ceux qui n'en ont pas besoin. C'est pourquoi le réveil des partisans du nucléaire auquel on assiste depuis la fin de 1991 en Égypte est lié au desserrement de sa contrainte financière et ne devrait donc être que conjoncturel. Une somme de 230 millions LE a été prévue dans le cadre du troisième plan quinquennal 1992-97 pour la construction d'un nouveau réacteur de recherche d'une puissance de $22 \mathrm{MW}$, pour relayer le réacteur soviétique de $2 \mathrm{MW}$ en service depuis 30 ans, qui sera construit par l'Argentine, aux termes d'un contrat signé en septembre 1992. Bien qu'aucun crédit pour la construction d'une centrale nucléaire ne soit prévu d'ici la fin 
de la décennie, l'EEA envisage une part de 7 \% pour l'électricité d'origine nucléaire en l'an 2005 (tableau 6), ce qui impliquerait de construire une centrale nucléaire d'environ $1.200 \mathrm{MW}$ durant les cinq premières années du prochain siècle, éventualité très hypothétique. La construction d'une usine de retraitement de déchets nucléaires liquides et d'une unité d'incinération de déchets radioactifs solides est par contre planifiée.

Tableau 6 : Évolution prévue de la répartition de la production d'électricité (\%)

\begin{tabular}{|l|c|r|r|}
\hline & $1990-91$ & 2005 & 2015 \\
\hline Hydro-électricité & 21,5 & 10 & 7 \\
Gaz naturet, fuel & 78,5 & 73 & 68 \\
Charbon, hydro électricité importée & - & 7 & 11 \\
Nucléaire & - & 7 & 9 \\
Energies nouvelles et renouvelables & - & 3 & 5 \\
Total & & 100 & 100 \\
\hline
\end{tabular}

Source : Egyplian Electricity Authority, octobre 1992.

37 L'utilisation de l'énergie solaire pourrait se baser sur l'importante radiation solaire dont bénéficie le territoire égyptien, qui varie de 6 à $8 \mathrm{KWh} / \mathrm{m} 2 /$ jour. Vu son coût, elle n'est prévue pour le moment que pour le chauffage de l'eau. Le développement de l'énergie éolienne est envisagé dans les régions de Marsa Matrouh et de Hurghada, l'objectif de l'Égypte étant une capacité installée de génération d'électricité éolienne de $70 \mathrm{MW}$, dont $40 \mathrm{MW}$ à l'issue du plan 1992-93/1996-97. Les perspectives d'emploi de l'énergie géothermique pour la génération d'électricité sont médiocres, vu la température relativement basse des sources repérées $\left(48^{\circ}\right.$ à $\left.78^{\circ}\right)$. L'usage domestique de la biomasse animale et végétale, qui fournissait environ le cinquième de la consommation égyptienne d'énergie en 1975, peut enfin s'accroître dans les campagnes, où elle tend cependant à être supplantée par le kérosène et le GPL. Au total, l'EEA prévoit que la part des énergies nouvelles et renouvelables dans la génération d'électricité progresserait à $3 \%$ du total en 2005, puis $5 \%$ en 2015. Ceci signifierait qu'à cette date, la production d'électricité issue de ce type de sources d'énergie atteindrait $8.160 \mathrm{GWh}$, ce qui suppose des avancées considérables dans ce domaine.

\section{D'ambitieux projets d'interconnexion électrique}

L'Égypte a récemment entrepris de connecter son réseau électrique à ceux de ses voisins. Le projet d'interconnexion électrique entre l'Égypte et la Libye doit être réalisé d'ici février 1994 pour un coût de 32 millions \$. Attribué à la société égyptienne Kahromica et financé par la Libye, le contrat porte sur l'installation de $170 \mathrm{~km}$ de câbles entre la ville libyenne de Tobrouk et le poste frontalier de Salloum, en Égypte. Dans une deuxième phase, ce dernier sera relié par une ligne de $450 \mathrm{~km}$ de long à la station de transformation égyptienne d'al-Amid. 
39 L'interconnexion égypto-jordanienne prévoit la pose d'une ligne $500 \mathrm{KV}$ de $276 \mathrm{~km}$ de long de Suez à Taba, où doivent être construites deux sous-stations de 500/220 KV et $400 \mathrm{KV}$, respectivement, et le franchissement du golfe d'Aqaba par un câble sous-marin de $400 \mathrm{KV}$ de $12 \mathrm{~km}$ de long, relié par une ligne aérienne de $10 \mathrm{~km}$ à la centrale thermique jordanienne d'Aqaba. Les appels d'offres pour les travaux sont en cours.

Les études de ce projet ont été confiées à la société française EDF International, de même que celles de l'interconnexion Égypte-Zaïre. Signé en juillet 1992 et financé par un don de 10 millions \$ de la Banque Africaine de Développement, ce dernier contrat doit être achevé en septembre 1994. Le projet porte sur/l'établissement d'une liaison entre les centrales hydro-électriques d'Assouan et d'Inga au Zaïre, pays dont la puissance hydro-électrique installée pourrait être portée de $1.700 \mathrm{MW}$ actuellement à 30.000 MW dans dix ans, puis 100.000 MW à terme. L'état anarchique actuel du Zaïre rend cette éventualité douteuse.

\section{De considérables investissements prévus}

41 L'augmentation annuelle de la demande, qui atteignait $10 \%$ au cours des deux précédentes décennies, devrait revenir à 5-6\% durant la décennie en cours, selon les prévisions de l'EEA. Malgré cela, l'EEA prévoit qu'entre les années 1991-92 et 2014-15 (tableau 7) la charge maximale appelée et la production d'électricité sur son réseau passeront respectivement de 7.215 MW à 26.620 MW (+269\%) et de 45.481 GWh à 163.205 GWh (+258,8\%). Selon la Banque mondiale, la capacité installée et la production d'électricité du réseau de l'EEA devraient augmenter au cours des huit années à venir de $32,6 \%$ et $47,1 \%$, passant respectivement à $15.453 \mathrm{MW}$ et $64.950 \mathrm{GWh}$ en 1998-99, soit une progression légèrement plus rapide (tableaux 8 et 9). Le plan 1992-93/1996-97 prévoit 36,5 milliards LE d'investissements dans le secteur de l'électricité, soit $25,2 \%$ des investissements planifiés, dont 21 milliards LE en devises. L'EEA prévoit quant à elle 43,4 milliards LE d'investissements de 1991-92 à 1998-99 (tableau 10), dont $72 \%$ en devises. Ce montant représente environ le tiers du produit intérieur brut de 1991-92, ce qui donne une idée de l'effort considérable prévu pour le secteur électrique, dont on peut se demander s'il est réaliste. 
Tableau 7 : Évolution prévue de la production d'électricité

\begin{tabular}{|c|c|c|c|c|}
\hline & $\begin{array}{c}\text { Charge } \\
\text { maximale } \\
\text { appelese (MW) }\end{array}$ & $\begin{array}{c}\% \\
\text { Varlation }\end{array}$ & $\begin{array}{l}\text { Production } \\
\text { d'electriclté } \\
\text { (Gwh) }\end{array}$ & $\begin{array}{c}\% \\
\text { Variation }\end{array}$ \\
\hline $1991-92$ & 7.215 & $\cdot$ & 45.481 & - \\
\hline 1992.93 & 7.610 & $+5,5$ & 47.638 & +4.7 \\
\hline $1993-94$ & 8.105 & +6.5 & 49.910 & +4.7 \\
\hline $1994-95$ & 8.490 & $+4,75$ & 52.140 & +4.5 \\
\hline $1995-96$ & 8.895 & $+4,8$ & 54.500 & +4.5 \\
\hline $1996-97$ & 9.360 & $+5,2$ & 57.225 & $+5,0$ \\
\hline 1997.98 & 9.940 & +6.2 & 60.550 & $+5,8$ \\
\hline 1998.99 & 10.590 & +6.5 & 64.320 & $+6,2$ \\
\hline $1999-2000$ & 11.345 & $+7,1$ & 68.720 & +6.8 \\
\hline $2000-01$ & 12.195 & $+7,5$ & 73.690 & $+7,2$ \\
\hline $2001-02$ & 13.110 & $+7,5$ & 79.065 & $+7,3$ \\
\hline 2002-03 & 13.485 & +2.9 & 84.415 & +6.8 \\
\hline 2003-04 & 14.620 & +8.4 & 89.385 & $+5,9$ \\
\hline 2004-05 & 15.440 & $+5,6$ & 94.590 & +5.8 \\
\hline $2005-06$ & 16.300 & +5.6 & 99.945 & +5.7 \\
\hline $2006-07$ & 17.215 & $+5,6$ & 105.540 & $+5,6$ \\
\hline 2007.08 & 18.180 & $+5,6$ & 111.450 & $+5,6$ \\
\hline $2008-09$ & 19.200 & $+5,6$ & 117.690 & +5.6 \\
\hline $2009-10$ & 20.270 & $+5,6$ & 124.285 & $+5,6$ \\
\hline $2010-11$ & 21.410 & $+5,6$ & 131.245 & $+5,6$ \\
\hline $2011-12$ & 22.600 & $+5,6$ & 138.595 & $+5,6$ \\
\hline $2012 \cdot 13$ & 23.870 & $+5,6$ & 146.355 & $+5,6$ \\
\hline 2013-14 & 25.210 & $+5,6$ & 154.550 & $+5,6$ \\
\hline 2014-15 & 26.620 & $+5,6$ & 163.205 & $+5,6$ \\
\hline
\end{tabular}

Source : Egyptian Electricity Authority, octobre 1992.

Tableau 8 : Évolution prévue du secteur électrique

\begin{tabular}{|c|c|c|c|c|c|c|c|c|}
\hline & 1991.92 & 1992.93 & $1993-94$ & 1994.95 & 1995.96 & $1996-97$ & $1997-98$ & 1998.99 \\
\hline Capacité installée (MW) & $\$ 1.690$ & 12.619 & 13.307 & 13.362 & 13.922 & 14.703 & 15.231 & 15.453 \\
\hline Production délectricité (GWh) & 46.739 & 49.120 & 51.637 & 54.338 & 56.872 & 59.519 & 62.234 & 64.950 \\
\hline dont : Hydro-électricité & 9.500 & 9.500 & 9.900 & 9.900 & 9.900 & 9.900 & 9.900 & 9.900 \\
\hline Turbines vapeur & 29.667 & 28.367 & 27.713 & 27.179 & 29.437 & 32.366 & 34.861 & 37.348 \\
\hline Turtines gaz & 7.191 & 10.689 & 13.358 & 16.473 & 16.606 & 16.780 & 16.928 & 17.076 \\
\hline Centrales isolèes & 381 & 564 & 666 & 786 & 929 & 473 & 545 & 626 \\
\hline $\begin{array}{l}\text { Consommation de gaz naturel } \\
\text { par les centrales }\left(\mathrm{Mm}^{3}\right)\end{array}$ & 4.763 & 5.354 & 5.873 & 6.498 & 6.766 & 7.084 & 7.347 & 7.607 \\
\hline
\end{tabular}

Source : Banque mondiale, mars 1992. 
Tableau 9 : Évolution prévue de la capacité Installée du réseau de l'EEA (MW)

\begin{tabular}{|c|c|c|c|c|c|c|}
\hline & 1990-91 & $\%$ & 1998-99 & $\%$ & \% variation & $\begin{array}{l}\text { Mise en service } \\
\text { prévus }\end{array}$ \\
\hline $\begin{array}{l}\text { Hydro-electricití } \\
\text { Haut barrage d'Assouan } \\
\text { Assouan I } \\
\text { Assouan " } \\
\text { Esna }\end{array}$ & $\begin{array}{r}2.715 \\
2.100 \\
345 \\
270\end{array}$ & 23,7 & $\begin{array}{r}2.815 \\
2.100 \\
345 \\
270 \\
100\end{array}$ & 18,2 & $+3,7$ & 1993.94 \\
\hline 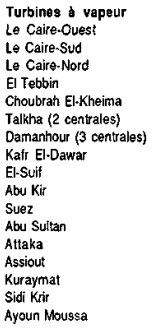 & $\mid \begin{array}{r}5.925,5 \\
350 \\
255 \\
100 \\
45 \\
1.295 \\
127.5 \\
525 \\
440 \\
113 \\
900 \\
185 \\
600 \\
900 \\
90\end{array}$ & 51,8 & \begin{tabular}{r|}
9.455 \\
950 \\
135 \\
$\vdots$ \\
1.260 \\
290 \\
525 \\
440 \\
60 \\
900 \\
165 \\
600 \\
900 \\
660 \\
1.260 \\
630 \\
630
\end{tabular} & 61,2 & $+59,6$ & $\begin{array}{l}1993.94 \\
1994.95 \\
1995.96 \\
1996.97 \\
1997.98\end{array}$ \\
\hline 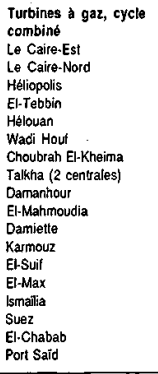 & $\begin{array}{r}2.676 \\
46 \\
330 \\
37,5 \\
46 \\
121 \\
100 \\
35 \\
283,5 \\
97 \\
376 \\
750 \\
25 \\
220 \\
28 \\
20 \\
17 \\
100 \\
44\end{array}$ & 23,4 & $\begin{array}{r}3.009,1 \\
495 \\
37.5 \\
46 \\
14 \\
100 \\
35 \\
283,6 \\
102 \\
284 \\
1.125 \\
220 \\
220 \\
20 \\
100\end{array}$ & 19,5 & $+12,4$ & 1993-94 \\
\hline Contrales isolées & 119 & 1,0 & 174 & 1,1 & $+46,2$ & \\
\hline Tolal & $11.435,5$ & 100 & $15.453,1$ & 100 & $+35,0$ & \\
\hline
\end{tabular}

Sources : EEA, Banque mondiale pour 1998-99.

Tableau 10 : Investissements prévus dans le secteur électrique(1991-92/1998-99)

\begin{tabular}{|l|r|r|}
\hline & Millions LE & \multicolumn{1}{|c|}{$\%$} \\
\hline Centrales électriques & $20.638,77$ & 47,5 \\
a) Projets en cours & & \\
Centrale cycle combiné Damiette (1.125 MW) & $2.999,18$ & \\
Centrale cycle combiné Caire-Sud (495 MW) & 394,74 & \\
Centrale cycle combiné Damanhour (142 MW) & 394,30 & \\
Cenirale cycle combiné Mahmoudia (284 MW) & 649,54 & \\
Extension centrale vapeur Talkha (290 MW) & 193,03 & \\
Extension centrale vapeur Assiout (690 MW) & $1.400,14$ & \\
Extension centrale vapeur Caire-Ouest (950 MW) & $1.770,86$ & \\
& & \\
b) Nouveaux projets & $15.639,59$ & \\
Cenirale vapeur Kuraymal (1.260 MW) & $5.079,79$ & \\
Centrale vapeur Sidi Krir (630 MW) & $3.273,92$ & \\
Centrale vapeur Ayoun Moussa (630 MW) & $3.271,04$ & \\
Aboukir, Damanhour et autres & 95,70 & \\
Centrale charbon Zaafarana & $3.919,14$ & \\
& & \\
Sous-stations électriques (66 à 500 KV) & $3.825,19$ & 8,8 \\
Lignes de transmission (66 à 500 KV) & $9.547,54$ & 22,0 \\
Frais financiers & $8.914,73$ & 20,6 \\
Autres dépenses (lormation, etc.) & 482,03 & 1,1 \\
Total & $43.408,26$ & 100,0 \\
\hline
\end{tabular}

- Puissances en fin de période.

Source : EEA 


\section{Conclusion}

42 La nouvelle politique énergétique égyptienne n'a pas tardé à produire les effets escomptés : la consommation de produits pétroliers a stagné dès 1991 et aurait baissé au premier semestre 1992, avec notamment une diminution de $4 \%$ de la consommation d'essence. Elle représente par ailleurs un instrument fondamental de la politique économique générale visant à réduire les subventions étatiques, parfois de manière indirecte. On peut par exemple remarquer que la hausse des prix de l'énergie renchérit l'usage des pompes agricoles, ce qui revient à faire payer l'eau d'irrigation, mesure essentielle de politique agricole, mais difficile à annoncer dans le contexte actuel... Cette augmentation pénalise par ailleurs les industries fortes consommatrices d'électricité - produits du mythe de la pléthore d'électricité apparu après la construction du haut-barrage - au premier rang desquelles la fonderie d'aluminium de Naga Hammadi et le complexe pétrochimique Kima, près d'Assouan, ce qui devrait provoquer des restructurations industrielles. Ces deux complexes industriels ont englouti à eux seuls $12 \%$ de la consommation égyptienne d'électricité en 1990-91, tout en ayant bénéficié des tarifs les plus bas ( $68,6 \%$ du prix moyen de gros). On peut noter que si un complexe pétrochimique peut être converti au gaz naturel, l'électricité est indispensable à la fonderie d'aluminium.

L'époque de l'énergie bon marché en Égypte est révolue. La hausse des prix de l'énergie entraînera une augmentation générale des prix, que l'économie égyptienne devra compenser par une amélioration de sa compétitivité. On mesure à présent combien les bas prix intérieurs de l'énergie ont entravé l'objectif économique d'amélioration de la productivité et ont faussé les décisions de production et de consommation. Aux effets pervers de la redistribution de la rente pétrolière, traditionnellement invoquée pour expliquer la difficulté des pays qui en sont dotés à bâtir une économie de production, il faut ajouter ces facteurs. À l'inverse, le relèvement des prix intérieurs de l'énergie et la modification de la nature de la rente pétrolière devraient contribuer à cette édification. Il apparaît en définitive que si, dans l'Égypte d'aujourd'hui, le principal enjeu de l'économie de l'énergie est l'économie d'énergie, cette dernière a des conséquences considérables sur l'ensemble de l'économie égyptienne.

\section{INDEX}

Mots-clés : économie, électricité, hydrocarbures, rente

\section{AUTEUR}

LOUIS BLIN

Cedej 